\title{
Wireless Sensor Network Clustering USING PARTIClES SWARM OPTIMIZATION FOR REDUCING ENERGY CONSUMPTION
}

\author{
Amin Rostami ${ }^{1}$ and Mohammad Hossin Mottar $^{2}$ \\ 1Department of Computer Engineering, Ferdows Branch, Islamic Azad University, \\ Ferdows , Iran. \\ 2Department of Computer Engineering, Mashhad Branch, Islamic Azad
}

\begin{abstract}
Wireless sensor networks (WSN) is composed of a large number of small nodes with limited functionality. The most important issue in this type of networks is energy constraints. In this area several researches have been done from which clustering is one of the most effective solutions. The goal of clustering is to divide network into sections each of which has a cluster head $(\mathrm{CH})$. The task of cluster heads collection, data aggregation and transmission to the base station is undertaken. In this paper, we introduce a new approach for clustering sensor networks based on Particle Swarm Optimization (PSO) algorithm using the optimal fitness function, which aims to extend network lifetime. The parameters used in this algorithm are residual energy density, the distance from the base station, intra-cluster distance from the cluster head. Simulation results show that the proposed method is more effective compared to protocols such as (LEACH, CHEF, $P S O-M V)$ in terms of network lifetime and energy consumption.
\end{abstract}

\section{Keywords}

Wireless sensor networks, clustering, Energy efficient protocols, Particles Swarm optimization algorithm, Centralized algorithms.

\section{Introduction}

Wireless Sensor Networks (WSN), as one of the most important technologies of the 21 century is discussed in this paper. These networks include many number of very small sensor nodes that are used for collection and peripheral information processing [1].Unlike ad hoc networks that may at the first glance very similar to the sensor networks, the nodes in the sensor network usually lack unique addresses and which is important for information collection by the sensors. Also due to lack of access to nodes after their disperse process, the network nodes are virtually useless and will die after that the available energy is over. So the energy consumption issue and optimization is one of the challenges raised in these networks. In recent years, many works have been done in this case [6]. Distinction between traditional telecommunication networks such as cellular systems and mobile ad hoc networks with WSN is that the networks have unique features such as: node density deployment, the lack of reliability of the sensor nodes and severe restrictions on energy computing and memory [3]. The applications that have already been proposed for sensor networks and are added day to day,can be refer to examples such as; routing in broad geographical environments, security system, control on large structures, control on patients with critical condition, control on environmental parameters in areas where human presence in the mis dangerous and so on [2]. In fact, Sensor networks is the accumulation of a large number of sensor nodes scattered in the environment. And each is autonomous and collaborated with other groups 
to follow a particular purpose. The nodes are close together and each node can communicate with other node and their data will be put to another node. Finally, under ambient conditions, the data is reported to a central node. Techniques and practices used in such networks highly depend on the nature of network usage and the network topology, atmospheric and environmental conditions, limitations and factors affecting network performance parameters and cost. So, today in the world wide valid universities and computer and electronics research centers, and especially Telecommunications, WSN, is a very attractive and popular field of research[4]. Many suggestions and researches are provided on various topics in this field.

The main goal of these efforts is to provide solutions with simple control method and low cost which finally lead to accountability toourneeds and can be persistent against limitations such as: bandwidth, energy, environmental interference, feeding and so on.

Also these solutions would be in accordance to our wishes and desires including the data transmission overload reduction, survivability and high lifetime and low cost. One of the challenges raised in the context of sensor networks is how to cluster nodes in the network since these networks be efficient in terms of available energy and processing resources [6].

Clustering sensor networks, is an effective technique to increase the scalability and survivability nodes, the main goal of clustering is to divide network to a set of individual and limited nodes that can be easy controlled. by apply clustering can routing table size, repeats end messages reiteration and energy consumption is reduced and enhance the network lifetime, until the nodes their data transfer to the shortest distance of associated cluster heads[9].

Indeed, proposed algorithm is a centralized algorithm. That all of the main decisions performed in base station. Such as: clustering and cluster head election. This algorithm make close itself to optimal mood in every time of performance, automatically and if occur erratic event in network, algorithm have this capability that decrease the effects of this problem to least quantity in whole of network, automatically and during shortest time. Also, because collecting data is centralized and do periodic, this algorithm is very suitable for when supervision and fined monitoring is needed by wireless sensor network.

Rest of the paper is organized as follows:
2.Related Works
3.Network model
4. The proposed method
5. Simulation
6. Conclusion

\section{RELATED WORKS}

LEACH $^{1}$ protocol [7] is the oldest part of the clustering algorithms in WSN which is a hierarchical method in which the routing method uses a single step. Its main objective is to maximize network lifetime, and distribute energy consumption across all network nodes. Nodes per cluster Received Information sent to cluster heads and The task of cluster heads is transmitted data to the base station. All data processing duties such as integration and collection is performed locally by cluster head. LEACH runs during several steps with two phases.

\footnotetext{
${ }^{1}$ Low Energy Adaptive Clustering Hierarchy
} 
1. Set up phase

2. Pitch phase

In the setup phase, each node decides the cluster heads or not. each node create a random number between 0 and 1 , that is, the probability of being selected as the cluster heads. If the probability $\mathbf{p}$ of node $\mathbf{n}$ is less than threshold $\mathbf{T}(\mathbf{n})$, the node $\mathbf{n}$ is a cluster head for current phase $\mathbf{r}$. In which $\mathbf{T}(\mathbf{n})$ is calculated as follow:

$t(n)= \begin{cases}\frac{p}{1-p *\left(r \bmod \frac{1}{p}\right)}, & \text { if } n \in G \\ 0 & \text { otherwise }\end{cases}$

Where $\mathrm{G}$ is the set of sensor nodes that are not in 1/P cluster heads of the final round. So in the first round 0 , each node of has the same probability $\mathrm{P}$ for clustering head., The amount of energy consumption during the network lifetime is effective and the algorithm is not efficient in terms of energy consumption.

In $\mathbf{C H E F}^{2}$ protocol [5] cluster head election mechanism uses fuzzy logic. CHEF algorithm obtains chance from two fuzzy set namely: residual energy of node and total distance between a node with other nodes that are located at a radius r. CHEF can overcome the defects of LEACH. This means that sensor node has more energy and has more chance of being cluster heads. the main advantages of CHEF can be summarized as follows:

1. Amount of computational overhead is minimized by using fuzzy logic.

Cluster heads are elected locally, that reduces the overhead associated with centralized processes. CHEF algorithm disadvantages can include:

1-CHEF algorithm employs few parameters in the election of cluster heads.

2-And the number of selected cluster heads greatly depends on the value of $\alpha$ :

In the TL-LEACH ${ }^{3}$ protocol [11]-It is an improvement in the basic LEACH algorithm.

The algorithm is performed in three steps:

1. $\mathrm{CH}$ selecting

2. Clusters formation

3. Data transmission

The algorithm uses two level cluster heads (primary and secondary).

Secondary cluster heads with other members which are located in one cluster communicate and after data aggregation transmit them to primary cluster heads and primary cluster heads route data to base station. The data which is sent to base station reduce energy consumption significantly but there is a lot of overhead in the election of primary and secondary cluster heads which affects the network lifetime.

EEHC ${ }^{4}$ Protocol [13] is a randomly distributed clustering algorithm. The EEHC algorithm assumes that communication environment is controversy and also there is an error-free environment. This algorithm is based on a two-stage clustering.

\footnotetext{
${ }^{2}$ Cluster Head Election mechanism using Fuzzy logic

${ }^{3}$ A Two -Levels Hierarchy for Low-Energy Adaptive Clustering Hierarchy

${ }^{4}$ Energy-efficient hierarchical clustering
} 
1. Single-level clustering

2. Multi-level clustering

Sensor nodes are cluster heads in hierarchical architecture. Cluster heads consume more energy than the other sensor nodes because, cluster heads have more work to do.

Hence, it is possible that the cluster heads energy is over faster than the other sensors. EEHC algorithm can be run periodically for load balancing or start to work when the cluster heads energy levels falls below a certain threshold. In Genetic Algorithm for Energy Efficient Clusters in Wireless Sensor Networks [12] fitness function calculation depends on the distance between cluster heads nodes and base station and the purpose of applying genetic algorithms is to elect the most appropriate cluster heads. It is a centralized method in which members of each cluster and sending time are determined. Each cluster head directly sends information to base station. Although this algorithm performs better than $\mathrm{LEACH}$, but there is not a significant improvement, because the computation of the fitness function is complex.

In the method of GA- MPI ${ }^{5}$,It is proposed [14]to divide the network into several clusters, but rather than sending data from nodes to cluster heads, they use a number of mobile agents in each cluster. The mobile agents perform the task of data collection from nodes, data aggregation sending them to the base station.

In this method, chromosomes are divided in two arrays:

1. Group array

2. Ordinal array

Group array includes a number of members of each cluster and ordinal array includes the nodes that belong to each cluster. Crossover only alters the nodes in the same group and the mutation alters the number of nodes in consistent group. In this method the purpose of using genetic algorithms is to calculate the optimal number of mobile agents and embellishment of clusters and is also network measurement using standard delay and does not consider the energy consumption. Simulations show that the sensor nodes reduce energy consumption by using mobile agents [20].

In the GFCM $^{6}$ protocol [21] which has actually improved FCM protocol for network clustering with combining genetic algorithm and fuzzy methods. Genetic algorithm is used to improve FCM method in clustering and the best cluster heads election. This method has the effectiveness in energy consumption balance of the network, which increases the network lifetime and reduces the energy consumption. Authors of [18] use PSO to create cluster in WSN and optimal clusters are sorted by fitness function based on within cluster distance. In this proposal, residual energy of nodes is ignored in the calculations, and fitness function is considered as follows:

$\sum_{j=1}^{k} \sum_{\mathrm{i}=1}^{\mathrm{n}_{\mathrm{j}}}\left(\mathrm{d}_{\mathrm{ij}}^{2}+\frac{\mathrm{D}_{\mathrm{j}}^{2}}{\mathrm{n}_{\mathrm{j}}}\right)$

Where $\mathbf{d}_{\mathbf{i j}}$ is distance between node $\mathbf{i}$ and cluster heads $\mathbf{j}$. $\mathbf{D}_{\mathbf{j}}$ is the distance between cluster heads $\mathbf{j}$ and base station and $\mathbf{n}_{\mathbf{j}}$ is the number of nodes in each cluster. In this way, inertia coefficient varies and the acceleration is constant. In [19] clustering wireless sensor networks based on improved discrete particle swarm optimization is proposed which tries to solve clustering inequality problem. In this method the number of nodes in each cluster is identical and the fitness function is only based on communication distance. In addition, PSO inertia coefficient is considered variable to distinguish between particles. Then the obtained cluster heads of PSO algorithm are checked in terms of energy levels and if their residual energy is less than a threshold value, the nearest node which its energy is higher than the threshold value will be

\footnotetext{
${ }^{5}$ Genetic algorithm based multiple MAs itinerary planning

${ }^{6}$ Genetic Fuzzy C-means algorithm
} 
replaced. This method is compared with the LEACH show better results, although the total residual energy of nodes and lifetime are not considered.

PSO-MV $\mathbf{M V}^{7}$ protocol [15] as well is based on PSO method, and since energy consumption in cluster heads is higher than other nodes, the purpose of the approach is energy balance. In the PSO-MV method, the 2 nodes are selected as cluster heads that is a node as main cluster heads $(\mathrm{MCH})$ and other as $(\mathrm{VCH})$ and the tasks between the nodes can be categorized, where the $\mathrm{MCH}$ is responsible for data collecting and transmission and $\mathrm{VCH}$ is responsible for inter-cluster communications or intra -cluster communications to BS. PSO-MV clustering algorithm is based on Routing of clusters included generation steps and transmission of data. Initialized for cluster head this is If the node of cluster heads candidate according to the set of nodes into clusters and threshold energy that shows to $\mathrm{E} \lambda$ is chosen as follow:

$\mathrm{E} \lambda^{\cdot}=\sum_{i=1}^{N} E i / N$

The simulation results show that date of node death in PSO-MV take more than clearly PSO, but the weakness of this algorithm is in selection the number of cluster heads optimum.

The $\mathbf{E E C S}^{\mathbf{8}}$ protocol [16] focuses to solve the problem of clusters distance from BS. In fact, the cluster which is far from the base station needs more energy consumption for data transmission. For this reason , of dynamic size to cluster is determined due to the distance of the cluster from base stations. This algorithm makes a more uniform distribution of energy in the whole network which leads to increasing network lifetime.

\section{Network Model}

- The nodes can use power control to adjust transmission power that is dependent on the distance to the receiver.

- The Sensor nodes are stationary.

- For each node a unique identity is assigned

- The nodes are aware of self-position. (via GPS)

- The nodes are homogeneous network, it means that have identical processing potency.

- The nodes have limited energy and after disperse there is no battery charging capabilities.

- Each node has an initial value which is $\mathbf{E}_{\max }$ and BS has no restrictions on energy, memory and communication.

- Links are symmetric that is two nodes $\mathrm{v}_{1}$ and $\mathrm{v}_{2}$ can use the same transmission power to connect.

\subsection{PSO clustering problem}

Two main problem of clustering using PSO method is the convergence to local optimal and slow convergence velocity, which is tried to be solved by using two ideas of chaos theory and acceleration strategy . In the formula of updating velocity of the cluster centers that is mentioned in the eq. (4) updating is done for each particle for relocating the particle to the new position, from the best answer for each particle (Pbest) and the best global solution so far (gbest). In

\footnotetext{
${ }^{7}$ Particle Swarm Optimization balanced energy consumption

${ }^{8}$ Energy-Efficient Cluster-Head Selection
} 
which $\mathbf{W}$ Inertia coefficient rate tends to previous velocity of the particle, $\mathrm{c}_{1}$ ratestends to the best local position of the particle, and $\mathrm{c}_{2}$ trendsto the best global position of the particle.

In eq. (5) replacing $c_{r}$ instead of $r_{r}$ improves PSO algorithm as given:

$$
\begin{aligned}
& v_{i d}^{\text {new }}=w \times v_{i d}^{\text {old }}+c_{1} \times r_{r} \times\left(\text { Pbest }_{i d}-x_{i d}^{\text {old }}\right)+c_{2} \times r_{2} \times\left(\text { gbest }_{i d}-x_{i d}^{\text {old }}\right) \\
& v_{i d}^{\text {new }}=w \times v_{i d}^{\text {old }}+c_{1} \times c_{r} \times\left(\text { Pbest }_{i d}-x_{i d}^{\text {old }}\right)+c_{2} \times\left(1-c_{r}\right) \times\left(\text { gbest }_{i d}-x_{i d}^{\text {old }}\right) \\
& C r_{t+1}=k \times C r_{(t)} \times\left(1-\operatorname{Cr}_{(t)}\right)
\end{aligned}
$$

In eq.(6), Crrandom value is created for each round independentlybetween 0and1.which substitutes bothr1andr2, and parameter $\mathrm{k}$ is the number of predicted clusters. Using the chaos theory in PSO population generation will result in more diverse of the algorithm.

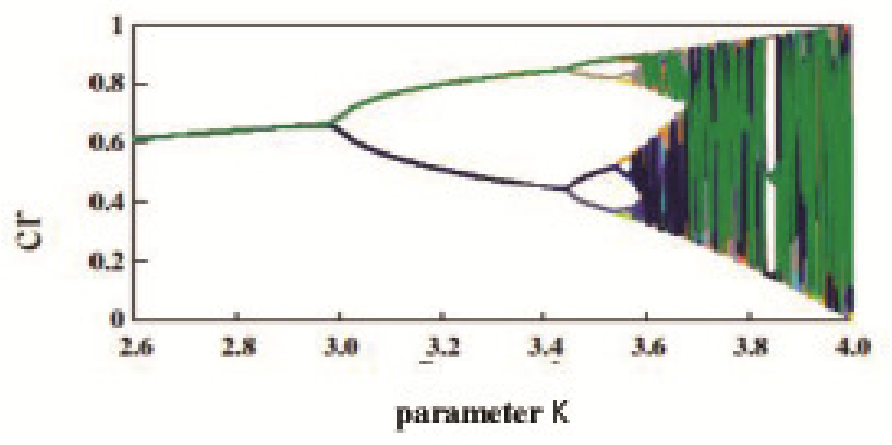

Figure1. Chaos map [17]

As can be see in Figure 1. To achieve more optimal particle swarm optimization algorithm, chaos theory is applied And in other change to increase the rate of convergence used acceleration strategy therefore in this mode a number of the population which are the best toward the target move not all population that it increases the rate of convergence [17].

\section{Proposed Method}

Our proposed algorithm is composed of two clustering and data transmission phases

\subsection{Clustering Phase}

In clustering phase, the particles are generated randomly. Then the best points are selected as the cluster heads and other nodes which are located near each cluster head becomes the member of the cluster and then fitness function is calculated for every cluster heads. If the fitness function is better than global best it is substituted. This process is donefor1000generation. Theneach node prepares a control message that contains identity and value of its residual energy and sends it directly to the base station. The base station which receives the information performs clustering operation. 


\subsection{Proposed Validation index}

As previously mentioned, the clustering is more desirable in which intra-cluster density is higher and in another word, the clusters are more cohesive and inter-cluster density is lower. Based on this principle, in the proposed method to estimate the optimal number of clusters. The first Select the number of clusters. Also to measure rate of clusters separation the different distance between cluster than total center of data set for the number of clusters considered, and then calculated the ratio between two, since the clustering is more desirable. The clusters are more compact and farther apart So, for the number of clusters where the index is maximum the clustering is more desirable and the optimal number of clusters is achieved. Validation index is composedoftwoparts,F1andF2:

validity $=\max (F 1+F 2)$

Whatever the amount of the above criterion is greater clustering is better. eq.(8) denotes the F1 index and Figure 3 illustrates the cluster dispersion and density of nodes in each cluster:

$\mathrm{F} 1=\left(\frac{\text { inter } \times 2}{\sqrt{\text { intra } \times 2}}\right)$

Inter: inter-cluster distance for which farther is better.

Intra: intra-cluster distance for which closer is better.

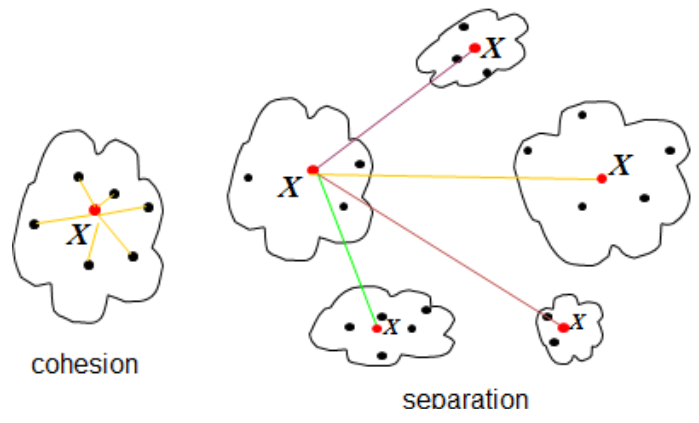

Figure2.Performance of the proposed index

Eq. (9),(10) denotes the intra and inter cluster separation:

$$
\operatorname{Intra}(\mathrm{c})=\sum_{i=1}^{c} \sum_{j=1}^{N}\left(X_{j}-X i\right)
$$

In Eq. (9) the total distance between nodes in each cluster and its cluster head ia calculated in which $\mathbf{c}$ is the number of clusters, $\mathbf{N}$ is the number of nodes, $\mathbf{X}_{\mathbf{j}}$ is the cluster head and $\mathbf{X i}$ denotes the distance of the nodes from its relative cluster head. The intra cluster separation is shown in the following equation:

$$
\operatorname{Inter}(\mathrm{c})=\sum_{j=1}^{c}(X j-X)
$$

To calculate the inter clusters separation, the distance between the centers of the clusters and the center of total data set is calculated. For cluster range specified the amount of this index calculate and show in chart. 
In the conditions in which the slope of the curve is sharper the estimate of the number of clusters is more accurate. Then with local search around the slope the optimal number of clusters can be reached. Eq. (11) Explains how to calculate F2.

$\mathrm{F} 2=$ cluster heads degree $+($ residual energy $\times 2) /$ centrality + distance to base station

Residual energy : because of the rest energy effect in being cluster heads is more effective we considered double its coefficients.

Cluster scale: the number of inter-cluster nodes divided by the total number of network nodes. Moreover in the above relationship (centrality)is obtained as follows:

centerality $=\frac{\sqrt{\frac{\sum d i s^{2}}{\mathrm{n}}}}{100}$

In which, $\sum d i s^{2}$ is the sum of squared distances of nodes to cluster heads. It is assumed that each node is aware of its position, and can calculate its distance from the base station.

F2 associated per experimental cluster heads to obtain and then its totalfor12experimental cluster heads is summed. Using 2 coefficient for energy is due to that in discussion of election the rest energy of cluster heads than other parameters have more important and is more effective and for a reason we are considered double its coefficient. In F1 formula without use of value coefficient, F1 than F2 was too small and invalidity could not significant effect so, we used morecoefficientsthatcouldbalancebetweenF1 andF2effect is created.

.As can be seen in this experiment, when the number of clusters change from 2 to16 the slope of our validity index change dramatically. Now with local search around the intervals, the exact number of clusters can be achieved.



Figure3. The proposed validity index when of the number of clusters change

\subsection{Data transmission phase}

After cluster formation and cluster heads election of each cluster; data can be transmitted by the normal nodes to corresponding cluster heads. In this phase, each normal node is connected to the nearest cluster head. Cluster heads are assigned with the implementation of a TDMA schedule to each cluster member. Each node in the allocated interval sends its data to cluster head in the form 
of data message. The cluster heads aggregate and transmit data towards base station after receiving all messages from cluster member nodes. Then the energy consumption of all nodes is computed.

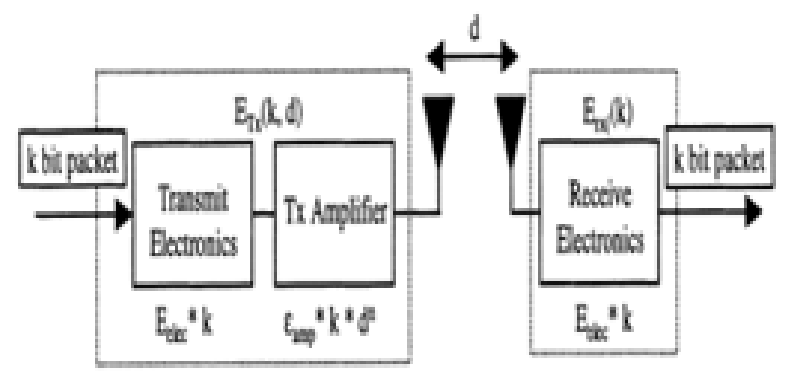

Figure4. Energy consumption model [7]

Energy model of the proposed protocol is the same as the energy model proposed in [7]and illustrated in Fig.4. Energy consumed per bit of data transfer on the distance $\mathbf{d}$ in Equation(13) is calculated as:

$E_{T X}(K, D)=\left\{\begin{array}{l}K \cdot E_{T X}(c d)=(k)+E_{T X_{a m p}}(k, d) \\ K \cdot E_{\text {elc }}(k, d)+k \cdot \varepsilon_{\text {friss }} d^{2} \text { if } d<d_{\text {crossover }}\end{array}\right.$

Energy consumption per $\mathrm{K}$ bit of data is calculated as follows:

$\operatorname{ERx}(k, d)=\operatorname{Erx} \_$elc $(k)=k$. Eelc

In the above equations, $\mathrm{E}_{\mathrm{elc}}$, denoted the energy of sending/receiving message size in terms of number of bits is denoted as $\mathrm{k}$, the distance between the transmitter and receiver is $\mathrm{d}$, the amplification energy is Etx_amp and $\mathcal{E}_{\text {friss }}$ amplifier factor and d crossover is threshold distance that transfer factor change in it. energy consumption of data gathering cluster heads is calculated as follows:

$E_{D A}=5 n j$. bit. $m s g$ 
International Journal of Managing Information Technology (IJMIT) Vol.6, No.4, November 2014

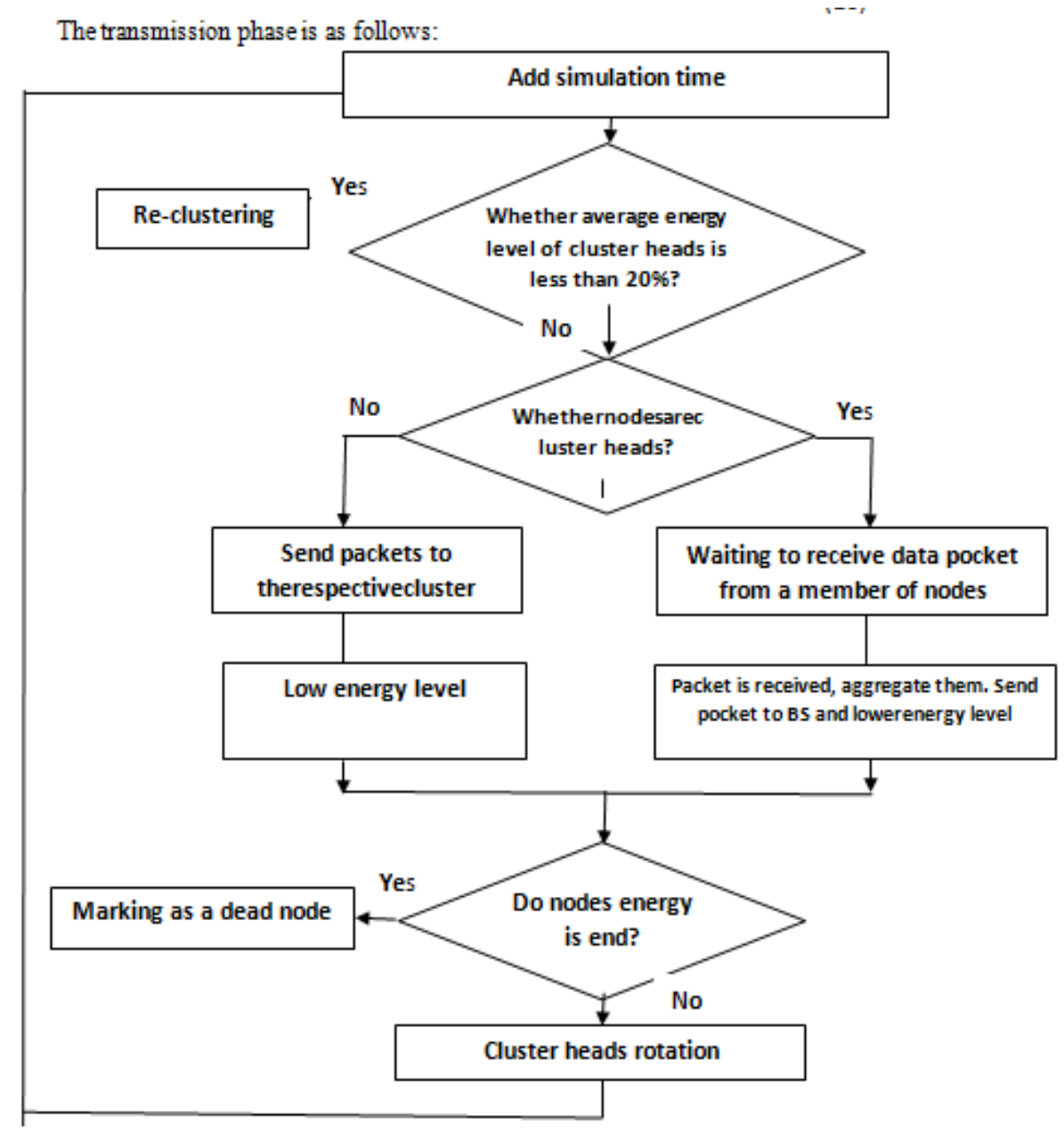

Figure 5. Data transmission phase flowchart

\section{Simulation}

The algorithm is simulated using MATLAB software. The parameters used are noted in the table 1. $\varepsilon m p$ and $\varepsilon f s$ are electronic energies, and EDA is the energy needed for data aggregation at cluster heads.

Table1.simulation parameters

\begin{tabular}{|l|l|}
\hline Parameter & \multicolumn{1}{c|}{ Value } \\
\hline$\varepsilon f s$ & $10 \mathrm{pJ} / \mathrm{bit} / \mathrm{m} 2$ \\
\hline$\varepsilon m p$ & $0.0013 \mathrm{pJ} / \mathrm{bit} / \mathrm{m} 4$ \\
\hline Eelec & $50 / \mathrm{nJ} / \mathrm{bit}$ \\
\hline EDA & $5 / \mathrm{nJ} / \mathrm{bit} /$ signal \\
\hline Initial energy per node & $0.5 \mathrm{j}$ \\
\hline Data packet size & $4000 \mathrm{bit}$ \\
\hline Control packet size & $200 \mathrm{bit}$ \\
\hline
\end{tabular}


The first step of our purpose approach is that using PSO to find the most optimal points in area and then the closest node to it are consider as cluster heads. we have a100 $\times 100$ area with 100 nodesrandomlydispersedand also the base station is put on the $50 \times 50$ coordinates. The number of particle and the velocity is calculated with respect to the area size .Initially, minimum particles together work is equal 20-bit and The velocity initially is equal 4.whichwascomparative and by increase the number of current nodes in the environment are changed.

The most influential parameters in the calculation of PSO are values that must be consider for $\mathbf{c 1}, \mathbf{c 2}, \mathbf{w}$, which in more papers are considered asc1 $=\mathbf{c 2}=\mathbf{2}$ and $\mathbf{w}=\mathbf{1}$. But to find more accurate values due to their significant impact on the problem solution, we evaluated all possible values between different intervals. After1000generations, with the cooperationof20particles together, as you can see in Figure 6thebest value for the parameter is equal to $\mathbf{c 1}=\mathbf{c} 2=0.5$ and $\mathbf{w}=0.007$.

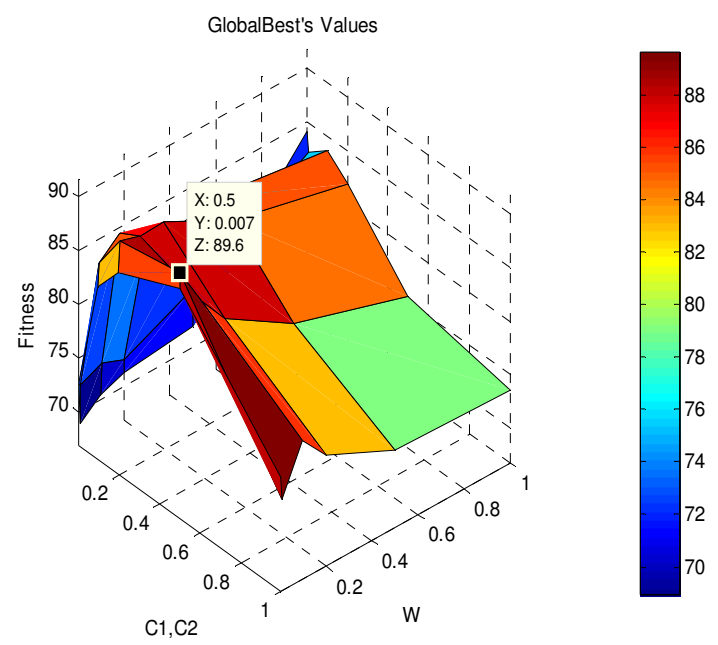

Figure6. The parameter values c1,c2,w

As the result of the random motion, the particles may be out of the environment that are required to move back into environment. We apply the support vector machine(SVM)supervised learning method to return the particle into the environment [22].

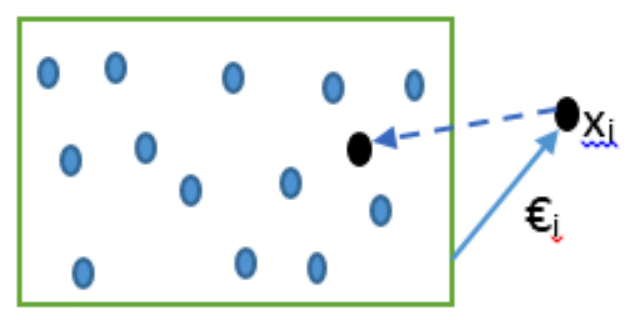

Figure7: represents a range of educational particles

In Fig 7 , there is a $X_{i}$ particles outside the range that with $\xi i\left(\xi_{i}>\mathbf{0}\right)$ is returned into the environment where compared to moving the particle on the border better results will be achieved. The next important issue is the value that should be considered for alpha. When the node energy is less than alpha value the cluster head is replacement. To consider the optimal alpha value all 
values between 0 and 1 with the distance of the 0.1 are considered. After running four times and averaging,the best alpha value for 100 nodes is equal to 0.8 and for 200 nodes is equal to 0.4whichcan be seen in Figure8.

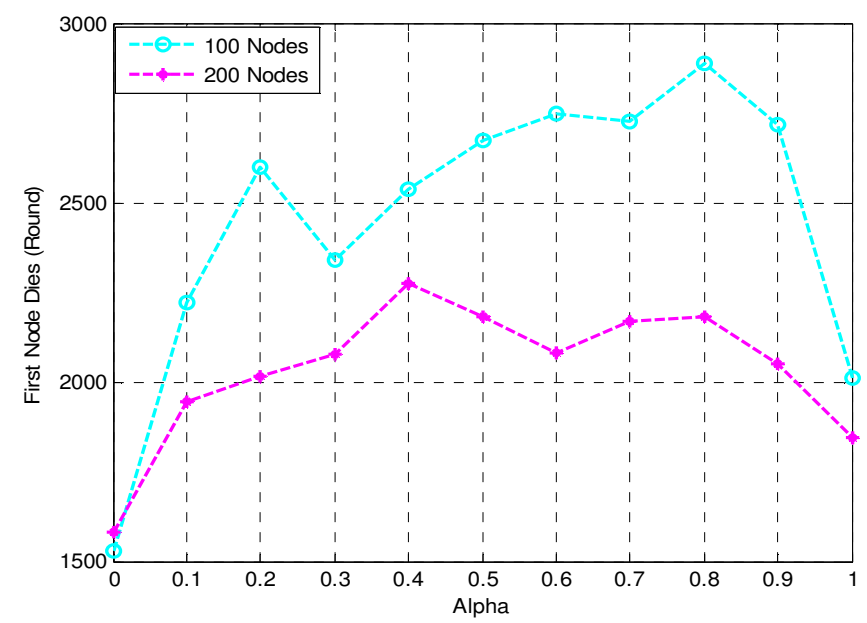

Figure 8. Alpha value in two cases of 100nodesand200nodes

Using the formula(11) and run it at 5000rpm for the best number of clusters is equal to 12 That at the end of the first node runs in 2959 round and half of them in 3682 round is dead.

As you can see in Figure9 that cluster heads are suitably dispersed. A point that should be noted is that the nodes that are close to the base station and its distance to the nearest cluster heads is less, transmit data directly to the base station and reduce energy consumption considerably.

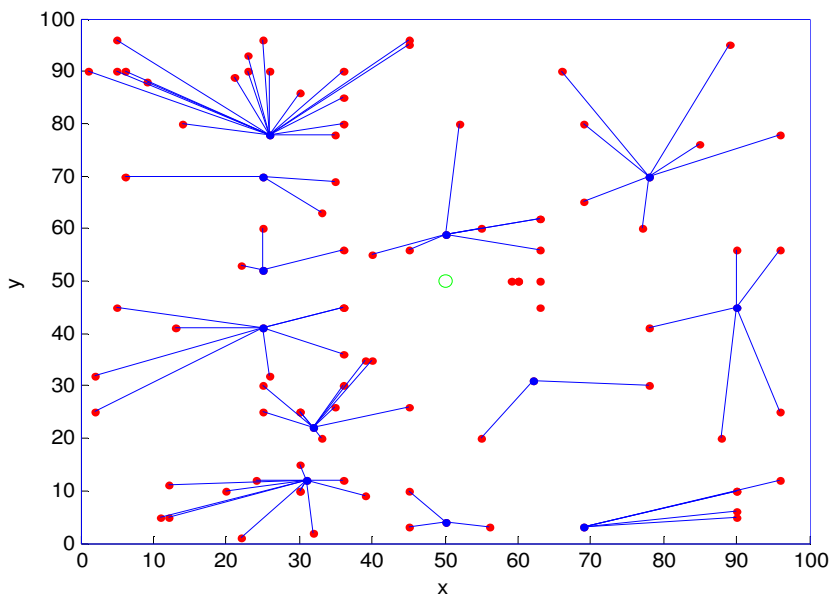

Figure9. The number of nodes associated to each cluster

We compared the proposed algorithm with the LEACH, CHEF, PSO-MV, GFCM algorithms which results are as follows. Figure 10shows the rate of dead nodes and network lifetime after implementing the proposed protocols which is higher compared to LEACH, CHEF, PSO-MV, GFCM protocols, which increases networks lifetime. 
International Journal of Managing Information Technology (IJMIT) Vol.6, No.4, November 2014



Figure 10. Comparing the proposed algorithm with four efficient algorithms, namely LEACH, CHEF, PSO-MV, GFCM in terms of the number of dead nodes

As shown in Figure 10 the first node in the LEACH algorithm dies at 790th round and the last dies at 1420th round, while using the proposed algorithm the first node dies in round2959 and the last node dies in the 4150the round which is due to the selection of the best possible cluster heads. Figure 11 denoted the energy consumed by LEACH, CHEF, PSO-MV, GFCM protocols and the proposed algorithm in which the proposed protocol has significantly lower total energy consumption than the other protocols.



Figure 11. Comparing the proposed algorithm with four efficient algorithms, namely LEACH, CHEF, PSO$\mathrm{MV}, \mathrm{GFCM}$ in terms of total energy consumption

As you can see in Figure11. the slope of the proposed algorithm is softer and suitable than that of the LEACH algorithm which lead to slower energy discharge. Therefore in LEACH 5\% of energy is lost in the 98th round and total energy is finished inthe1238th round While using the proposed algorithm, 5\% of energy is lost inthe233rd round and total energy is finished in the 3971 stround, which increases of network lifetime.

\section{CONCLUSIONS}

In this paper, we introduce a new approach for sensor network clustering using Particle Swarm Optimization (PSO) algorithm. The parameters which are used in the algorithm are residual 
International Journal of Managing Information Technology (IJMIT) Vol.6, No.4, November 2014

energy, density, distance from the base station, intra-cluster distance and cluster heads distance from each other. Our goal was to propose a new cost function to select the best cluster heads that combine the various criteria affecting the energy efficiency of cluster heads and cluster heads rotation among the nodes. Also, using the proposed algorithm the network coverage is evaluated and compared with some previous methods which have proved better performance and improved network lifetime and energy consumption.

\section{REFERENCES}

[1] Akiyldiz, W. Su, Y. Sankarasubramaniam, E. Cayirci, A survey on sensor networks, IEEE Communication Magazine, vol 40, 2002,pp 102-114.

[2] G. Pottie and W. Kaiser, Wireless integrated sensor networks(WINS), Communications of the ACM, vol. 43 , no. 5 , 2000,pp. 55-58.

[3] G.Hill, R. Szeweyk, A. Woo, D. Culler, S. Hollar, and K.Pister,System architecture directionsfor networked sensors, in proceedings of the International Conference on Architectural Supportfor programming Languages and Operating Systems (ASPLoS IX). Cambridge, MA, 2000,pp. 93-104.

[4] W. Merrill, K. Sohrabi, L. Girod , j. Elson , F. Newberg , and W. Kaiser, Open standard development plarroems for distributed sensor networks, in Proceedings of SPIE Unattended Ground Sensor Technologies and Applications IV, Orlando, FL.2002,pp,327-337.

[5] J. M. Kim, S.H. Park, Y.J. Han, T.M. Chung, CHEF: cluster head election mechanism using fuzzy logic in Wireless Sensor Networks, in: International Conference of Advanced Communication Technology, 2008, pp. 654-659.

[6] N.Eghbali, Performance Improvement of Information Dissemination Protocols in Sensor Networks Through Data Aggregation, Computer Engineering and Information TechnologyDepartment, 2007. PP 1-121.

[7] W. Heinzelman, A. Chandrakasan, H. Balakrishnan, Energy Efficient Communication Protocol forWireless MicrosensorNetwroks (LEACH), Proceedings of 33rd hawaii international conferencesystems science - vol.8,2004, pp 3005-3014.

[8] M. Handy , M. Haase , and D.Timmermann, Low energy adaptive clustering hierarchy with deterministic cluster - head selection, in proceeding of IEEE, International Conference on Mobile and wirless Communications networks, Stockholm, Sweden,2002, pp. 368-372.

[9] V. Handziski, A. Kopke, H. Karl, C. Frank, W. Drytkiewicz, Improving the Energy Efficiency of Directed Diffusion Using Passive Clustering," European Workshop on Wireless SensorNetworks 2004 , pp. 172-187.

[10] O.Younis and S.Fahmy,HEED:Ahybrid,energyefficient,distributed clusteringapproach for adhoc sensor networks,IEEE Transactions on Mobile Computing,vol.3,no.4,2004,pp.366-379.

[11] V. Loscrì, G. Morabito, S. Marano, A Two-Levels Hierarchy for Low-Energy AdaptiveClustering Hierarchy, in proceeding of IEEE 2005,pp. 1809-1813.

[12] S. Hussain, A.W. Matin, and O. Islam. Genetic algorithm for energy efficient clusters in wireless sensor networks. In Fourth International Conference on Information Technology,2007,pp147-154.

[13] D.kumar, T.C.Aseri and R.B.Patel EEHC: Energy efficient hetergenous clustered scheme for wireless sensor networks, ComputerCommunications, vol 32,2009,pp.662-667.

[14] K. LingaRaj, D. Aradhana and NagaveniB.Biradar. Multiple mobile agents in wireless sensor networks using genetic algorithms. International Journal of Scientific and Engineering Research, vol3,2012, pp1-5.

[15] H.Yua, W. Xiaohuia PSO-based Energy-balanced Double Cluster-heads Clustering Routing for wireless sensor networks ,Procedia Engineering, vol 15 ,2011, pp.3073 - 3077.

[16] S.-H. Hong, B.-K. Kim and J.-M. Gil An Algorithm for an Energy-Efficient Smart Sensor with EECS Routing Protocol in Wireless Sensor Networks, Springer Science Business Media Dordrecht 2012, pp. 685-692.

[17] Li-Yeh Chuang a, Chih-Jen Hsiao b, Cheng-Hong Yang, Chaotic particle swarm optimization for data clustering,Elsevier, 2011. PP 109-115.

[18] S.M. Guru, S.K. Halgamuge, and S. Fernando. Particle swarm optimisers for cluster formation in wireless sensor networks. In Proceedings of the International Conference on Intelligent Sensors, Sensor Networks and Information Processing,2005,pp 319-324.

[19]J. Hou, X. Fan, W. Wang, J.Jie, and Y. Wang. Clustering strategy of wireless sensor networks 
International Journal of Managing Information Technology (IJMIT) Vol.6, No.4, November 2014

based on improved discrete particle swarm optimization.In The Sixth International Conference on NaturalComputation (ICNC), vol 7,2010,pp3866-3870.

[20] B.Solaiman , A. Sheta, Computational Intelligence for Wireless Sensor Networks: Applications and Clustering Algorithms,International Journal of Computer Applications ,Vol 73 ,2013, pp0975 - 8887.

[21] S . He , Y, Dai , R , Zhun , S , Zhao , A Clustering Routing Protocol for Energy Balanc of WSN based on Genetic Clustering Algorithm , International Conference on Future Computer Supported Eduction ,2012, pp 788-793

[22] J.Weston and C. Watkins, Multi-class support vector machines, Technical Report CSDTRDepartment of Computer Science, Royal Holloway, University of London,Egham, 1998, pp98-104. 